\title{
Синтез нанокомпозита гидроксид хрома (III) - катионообменник КУ-2-8
}

\author{
(C) 2020 Козадерова О.А. \\ Воронежский государственный университет инженерных технологий, Воронеж \\ Поступила в редакцию 02.11.2020 г.
}

DOI: $10.17308 /$ sorpchrom.2020.20/3053

Работа посвящена получению нанокомпозита гидроксид хрома (III) - катионообменник посредством включения $\mathrm{Cr}(\mathrm{OH})_{3}$ в матрицу сильнокислотного катионита. Гидроксидсодержащий гибридный катионообменник синтезировали на основе полимерного носителя с сульфоновокислыми ионообменными группами КУ-2-8, поэтапно переводя функциональные группы из натриевой в $\mathrm{Cr}^{3+}$ форму и осаждая гидроксид хрома (III) в зерно катионообменника раствором щелочи $\mathrm{NaOH}$. Coдержание хрома в нанокомпозите определяли по концентрации вышедших в раствор противоионов методом прямой потенциометрии. Морфологию поверхности гибридных хром-полимерных гранул исследовали методом сканирующей электронной микроскопии. Элементный анализ состава скола нанокомпозита определяли методом рентгеновского энергодисперсионного микроанализа. Найдено, что поверхность гибридной гранулы однородна. Сера и натрий достаточно равномерно распределены по всему объему полимерных гранул за исключением внешнего слоя, обедненного этими элементами. Хром обнаружен не только в поверхностном слое гранулы толщиной 10-15 мкм, но и в объеме зерна, где его концентрация существенно ниже. Распределение хрома по объему зерна является неравномерным. Показано, что при переходе от периферии зерна к центру концентрация хрома экспоненциально снижается. Элементное картирование подтверждает, что хром находится в грануле катионообменника в окисленной форме. Предложен механизм формирования нанокомпозита гидроксид хрома (III) - катионообменник КУ-2-8 с сульфоновокислыми ионообменными группами. В ходе реакции ионообменного насыщения происходит переход ионита в $\mathrm{Cr}^{3+}$-форму. При подщелачивании реакционной среды осадок гидроксида хрома (III) локализуется вблизи поверхности катионообменника. Формирование осадка $\mathrm{Cr}(\mathrm{OH})_{3}$ в наноразмерных порах катионита препятствует диффузионному переносу ионов $\mathrm{Cr}^{3+}$ по направлению к его поверхности, в то время как Доннановское исключение не позволяет гидроксильным ионам в достаточной концентрации проникнуть достаточно глубоко в объем гранулы ионита. Показана возможность получения наночастиц модификатора (гидроксида хрома (III)) с неравномерным распределением в объеме гранулы, объединенных в агломераты микрометрового размера.

Ключевые слова: нанокомпозит, катионообменник КУ-2-8, гидроксид хрома (III)

\section{Введение}

В настоящее время все больше исследований направлено на получение гибридных нанокомпозитных материалов ионообменник-металл, что открывает новые возможности для ионообменников, которые после модификации способны выступать в роли электроноионообменников и применяться в каталитических, электрокаталитических и сорбционных процессах [1-7]. При этом ионообменники, характеризующиеся пористостью наноразмерного масштаба, способны выступать не только в роли нанореакторов, предотвращая агломерацию нанодисперсных частиц, что необходимо для обеспечения большой площади контакта между реагентами в процессах сорбции, но и использовать его в качестве поддерживающего материала. Например, 
введение в анионообменные гранулы гидроксидов переходных металлов, выступающих в роли сорбентов и применяемых для очистки воды, позволит использовать закрепленный на носителе сорбент в составе элементов колонного типа. Преимуществом таких гибридных материалов по сравнению с оксидами металлов, синтезируемыми обычно в форме суспензий, является отсутствие проблемы возможного загрязнения очищенной воды наноструктурированными сорбентами. Это открывает новые возможности аппаратурного оформления сорбционной очистки воды. Еще одним преимуществом закрепления наносорбентов на полимерном носителе является получение «аккумулированной» формы наночастиц, что упрощает процедуру их применения и утилизации $[8,9]$.

Катализаторы на основе оксида хрома (III) применяются для газофазного окисления $\mathrm{HCl}$ [10]. Гидроксид хрома (III), обладает наибольшей (по сравнению с другими d-металлами) каталитической активностью при разложении молекул воды на границе катионо- и аноинообменного слоев биполярной мембраны $[11,12]$. В связи с этим актуальной задачей является исследование закономерностей распределения хрома в сульфокатионообменнике (для применения в самостоятельной форме или в составе ионообменной мембраны).

Цель настоящего краткого сообщения - получение нанокомпозита гидроксид хрома (III) - катионообменник включением $\mathrm{Cr}(\mathrm{OH})_{3}$ в матрицу сильнокислотного катионита.

\section{Экспериментальная часть}

Синтез гидроксидсодержащего гибридного катионообменника проводили на основе полимерного носителя с сульфоновокислыми ионообменными группами КУ2-8. Неорганический осадок $\mathrm{Cr}(\mathrm{OH})_{3}$ вводили в катионит в два этапа. Прежде всего, функциональные группы количественно переводились из натриевой в $\mathrm{Cr}^{3+}$-форму, после чего раствором щелочи осаждали гидроксид хрома (III) в зерно катионообменника. На первом этапе катионит в $\mathrm{Na}^{+}$-форме $\left(\mathrm{R}_{-} \mathrm{SO}_{3}{ }^{-} \mathrm{Na}^{+}\right)$массой $\sim 1.0$ г помещали в коническую колбу и обрабатывали раствором $\mathrm{Cr}_{2}\left(\mathrm{SO}_{4}\right)_{3}\left(50 \mathrm{~cm}^{3}, \mathrm{c}\left(1 / 3 \mathrm{Cr}^{3+}\right)=\right.$ 0.25 моль/дм $\left.{ }^{3}\right)$ при $20^{\circ} \mathrm{C}$ в течение 1 часа при непрерывном перемешивании. На втором этапе отфильтрованный промежуточный продукт помещали в раствор $\mathrm{NaOH}$ $\left(50 \mathrm{~cm}^{3}, \mathrm{c}=0.1\right.$ моль/дм $\left.{ }^{3}\right)$. Содержание хрома в конечном продукте определяли, отслеживая концентрацию вышедших в раствор противоионов методом прямой потенциометрии непосредственно во время протекания реакции насыщения. Морфологию поверхности гибридных хром-полимерных гранул исследовали с применением сканирующей электронной микроскопии на растровом электронном микроскопе с катодом полевой эмиссии Quanta Nova (FEI, Голландия) в режиме вторичных электронов. Элементный анализ состава скола гранулы выполняли на приставке энергодисперсионного анализа EDAX (США).

\section{Обсуждение результатов}

В качестве исходного материала для осаждения гидроксида хрома (III) использовался КУ-2-8 - сильнокислотный катионит, содержащий сульфоновокислые группы (в количестве 4.0 мг-экв $\Gamma^{-1}$ сухой смолы). Осадок был введен в матрицу в результате следующих реакций:

- ионообменного насыщения:

$3 \mathrm{R}-\mathrm{SO}_{3}{ }^{-} \mathrm{Na}^{+}+1 / 2 \mathrm{Cr}_{2}\left(\mathrm{SO}_{4}\right)_{3}=\left(\mathrm{R}-\mathrm{SO}_{3}{ }^{-}\right)_{3} \mathrm{Cr}^{3+}+3 / 2 \mathrm{Na}_{2} \mathrm{SO}_{4}$;

Козадерова О.А. / Сорбционные и хроматографические процессы. 2020. Т. 20. № 5. С. 608-614 
- образования труднорастворимого $\mathrm{Cr}(\mathrm{OH})_{3}$ :

$\left(\mathrm{R}-\mathrm{SO}_{3}{ }^{-}\right)_{3} \mathrm{Cr}^{3+}+3 \mathrm{NaOH}=\left[\mathrm{R}-\mathrm{SO}_{3}{ }^{-} \mathrm{Na}^{+}\right]_{3} \cdot \mathrm{Cr}(\mathrm{OH})_{3}$.

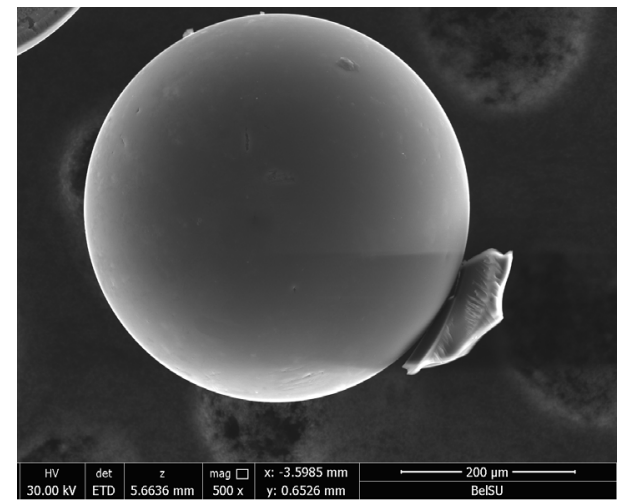

Рис. 1. СЭМ-изображение поверхности гранулы нанокомпозитного катионита

Fig. 1. SEM image of the surface of a nanocomposite cation exchanger granule

Как видно из рис. 1, поверхность гибридной гранулы однородна. Микрофотографии СЭМ скола гранулы (рис. 2) показывают, что она имеет полимерную сердцевину, в которой равномерно распределены небольшие кристаллические включения разного размера в зависимости от степени удаления с поверхности вглубь гранулы, и внешний крупнокристаллический слой толщиной 10-15 мкм. В нашей работе [13] было показано, что гидроксид хрома(III) при введении в сульфокатионообменник находится в наноразмерных порах и каналах ионита, входящего в состав мембраны, а определяемые микроскопически Cr-содержащие частицы модификатора представляют собой конгломераты наночастиц с размером 10-40 нм. По всей видимости, аналогичная ситуация наблюдается в данном случае (условия введения гидроксида хрома (III) в КУ-2-8 в настоящей работе и в мембрану МБ-2 в работе [13] идентичны).
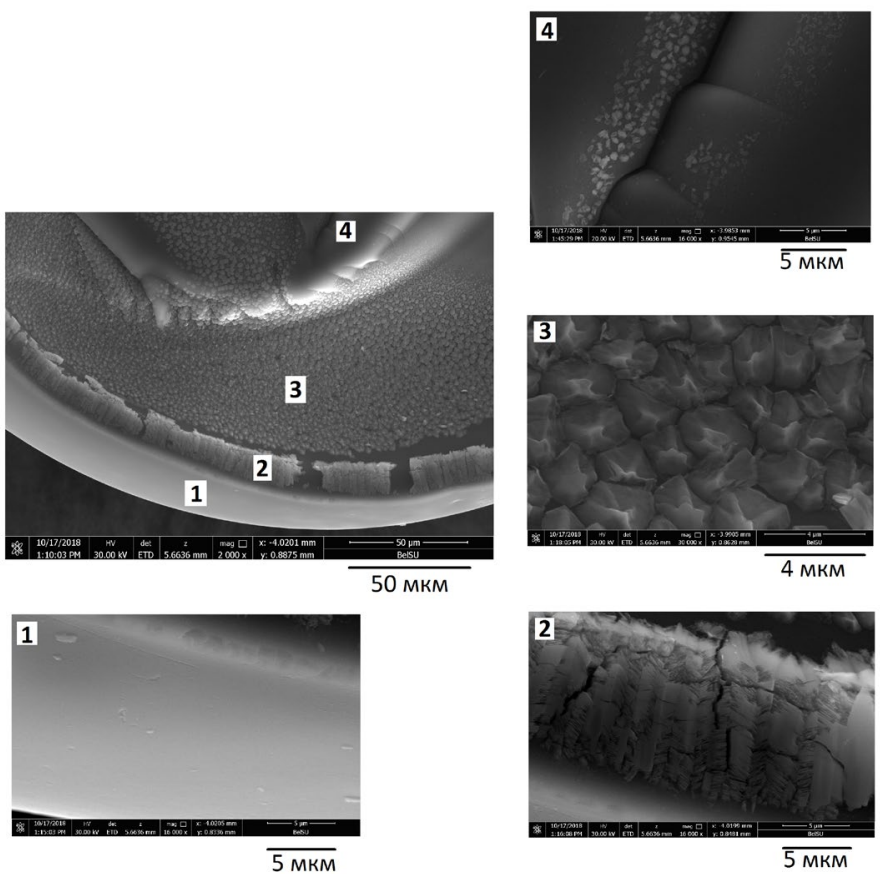

Рис. 2. СЭМ-изображения скола полученных гранул нанокомпозитного катионита

Fig. 2. SEM images of the cleavage of the obtained granules of the nanocomposite cation exchanger 
На рис. 3 представлены изображения, показывающие элементный состав скола гранулы полученного гибридного полимера, что позволило проанализировать содержание $\mathrm{O}, \mathrm{Cr}, \mathrm{S}$ и $\mathrm{Na}$ в разных частях катионита. Результаты показали, что сера и натрий достаточно равномерно распределены по всему объему полимерных гранул за исключением внешнего слоя, обедненного этими элементами. Хром находится в непосредственной близости от оболочки гранулы, а также на внешней поверхности сферической частицы. Элементная карта $\mathrm{Cr}$ для исследуемых образцов показывает, что хром обнаружен не только в поверхностном слое гранулы толщиной 10-15 мкм, но и в объеме зерна. Однако его объемное содержание намного меньше, а распределение по объему зерна является неравномерным: при удалении от поверхности в глубь гранулы содержание хрома уменьшается. Кислородная карта совпадает с распределением хрома на сколе гранулы, что служит свидетельством того, что хром находится в окисленной форме. Количественный анализ относительного содержания хрома в зерне позволил получить графическую зависимость распределения металла в грануле в радиальном направлении (рис. 4). Видно, что при переходе от периферии зерна к центру концентрация хрома экспоненциально снижается.
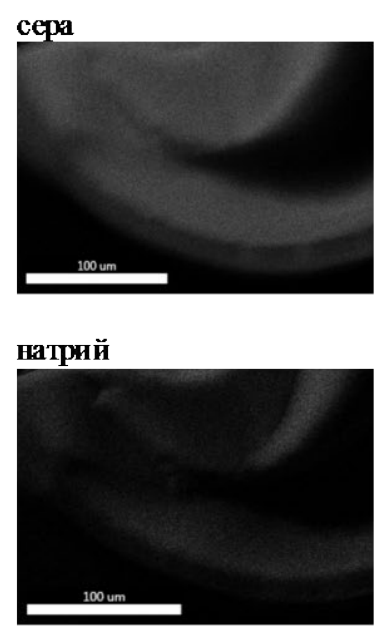
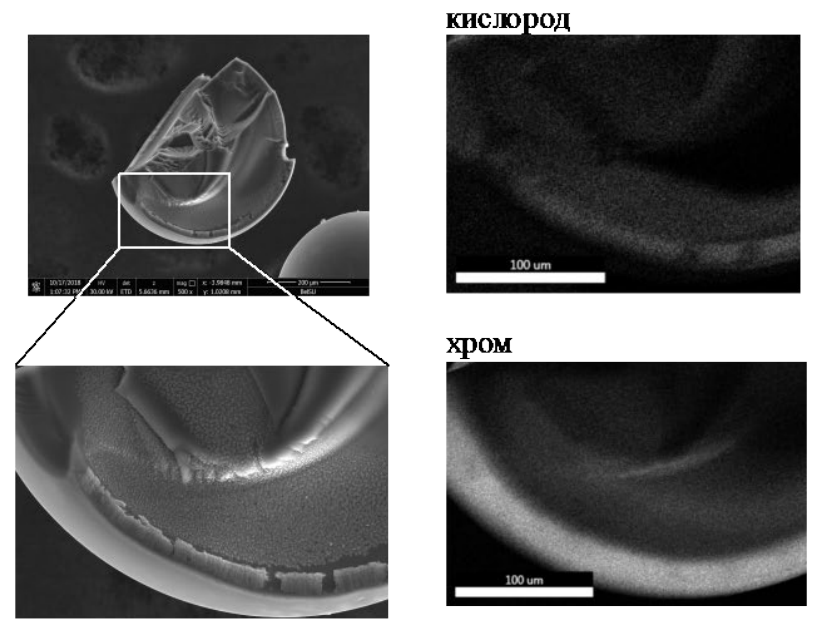

Рис. 3. Карты распределения элементов $\mathrm{S}, \mathrm{Na}, \mathrm{O}$ и $\mathrm{Cr}$, зарегистрированные на сколах полученных гибридных полимерных гранул

Fig. 3. Distribution maps of $\mathrm{S}, \mathrm{Na}, \mathrm{O}$, and $\mathrm{Cr}$ elements recorded on cleavages of the obtained hybrid polymer granules

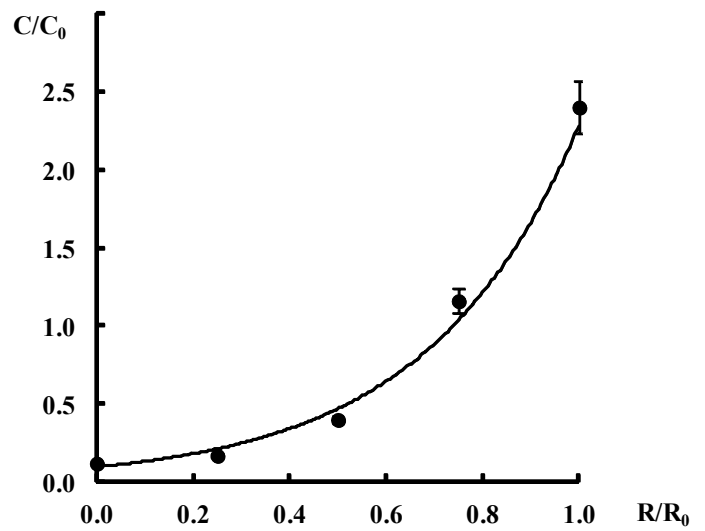

Рис. 4. Зависимость относительной концентрации хрома

(по отношению к обменной емкости) от радиуса зерна сульфокатионообменника

Fig. 4. Dependence of the relative concentration of chromium(in relation

to the exchange capacity) on the radius of the grain of the sulphonic cation exchanger 


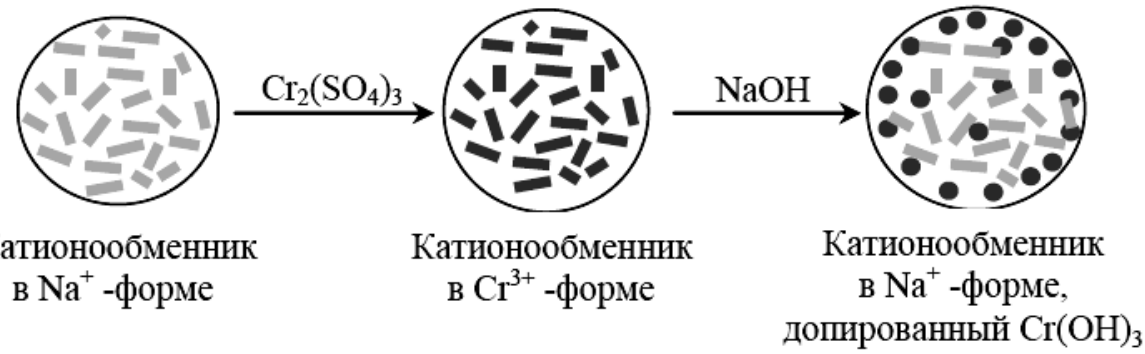

Рис. 5. Формирование нанокомпозита гидроксид хрома (III) катионообменник КУ-2-8

Fig. 5. Formation of nanocomposite chromium (III) hydroxide KU-2-8 cation exchanger

Описанные выше особенности взаимодействия хрома и сульфокатионообменника позволяют предложить следующий механизм формирования нанокомпозита гидроксид хрома (III) - катионообменник КУ-2-8 для рассмотренных условий ситеза (рис. 5). При реакции ионообменного насыщения ионит переходит в $\mathrm{Cr}^{3+}$-форму. При подщелачивании реакционной среды осадок гидроксида хрома (III) локализуется вблизи поверхности. $\mathrm{Cr}(\mathrm{OH})_{3}$, осажденный в порах катионита, препятствует диффузии ионов $\mathrm{Cr}^{3+}$ к поверхности, а Доннановское исключение не позволяет гидроксильным ионам в достаточной концентрации проникнуть глубоко в гранулу.

\section{Заключение}

Гидроксид хрома (III) может быть внедрен в матрицу сульфокатионообменника КУ-2-8 путем последовательной обработки катионита солью хрома (III) и раствором щелочи. Полученный гибридный полимер характеризуется градиентным распределением хрома: он сосредоточен преимущественно вблизи поверхности зерна (слой толщиной 10-15 мкм), при перемещении от поверхности вглубь зерна его концентрация экспоненциально падает. Таким образом, показана возможность получения наночастиц модификатора (гидроксида хрома (III)) с неравномерным распределением в объеме гранулы, объединенных в агломераты микрометрового размера.

\section{Список литературы}

1. Кравченко Т.А., Калиничев А.И., Полянский Л.Н. Нанокомпозиты металлионообменник. М. Наука. 2009. 391 с.

2. Huang M., Shen Y., Cheng W., Shao Y. et al. // Anal. Chim. Acta. 2005. Vol. 535. Is. 1-2, pp. 5-22.

3. Camargo P.H.C., Satyanarayana K.G., Wypych F. // Mater. Res. 2009. Vol. 12. No 1. pp. 1-39.

4. Domènech B., Bastos-Arrieta J., Alonso A., Macanás J., Muñoz M., Muraviev D.N. Bifunctional Polymer-Metal Nanocomposite Ion Exchange Materials, Ion Exchange Technologies, Ayben Kilislioğlu, IntechOpen, DOI: 10.5772/51579. Режим доступа: https://www.intechopen.com/books/ion- exchange-technologies/bifunctional-polymermetal-nanocomposite-ion-exchange-materials

5. Юрова П.А., Караванова Ю.А., Стенина И.А., Ярославцев А.Б. // Российские нанотехнологии. 2016. Т. 11. № 11-12. С. 75-78.

6. Сафронова Е.Ю., Паршина А.В., Янкина К.Ю., Рыжкова Е.А. и др. // Мембраны и мембранные технологии. 2017. Т. 7. № 2. С. 110-116.

7. Крысанов В.А., Плотникова Н.В., Кравченко Т.А. // Журн. физич. химии. 2018. Т. 92. № 3. C. 434-438.

8. Wang D., Lin Z., Wang T., Yao Z. et al. // J. Hazard. Mater. 2016. Vol. 308. pp. 328-344.

9. Su C. // J. Hazard. Mater. 2017. Vol. 322. pp. $48-84$. 
10. Amrute A.P., Mondelli C., Perez-Ramirez J. // Catalysis Science \& Technology. 2012. Vol. 2. pp. 2057-2065.

11. Шельдешов Н.В., Заболоцкий В.И., Ганыч В.В. // Электрохимия. 1994. Т. 30. № 12. C. $1458-1461$.
12. Мельников С.С., Шаповалова О.В., Шельдешов Н.В., Заболоцкий В.И. // Мембраны и мембранные технологии. 2011. Т. 1. № 2. C. 149-156.

13. Kozaderova O.A. // Nanotechnologies in Russia. 2018. Vol. 13. pp. 508-515.

\title{
Synthesis of a chromium (III) hydroxide nanocomposite - $\mathrm{KU}-2-8$ cation exchanger
}

\author{
(C) 2020 Kozaderova O.A. \\ Voronezh State University of Engineering Technologies, Voronezh, Russian Federation
}

\begin{abstract}
The study is devoted to the preparation of a chromium (III) hydroxide nanocomposite - cation exchanger by the inclusion of $\mathrm{Cr}(\mathrm{OH})_{3}$ into the matrix of a strongly acidic cation exchanger. A hydroxidecontaining hybrid cation exchanger was synthesized based on polymer carrier with KU-2-8 sulphonic acid ion-exchange groups, step by step transferring functional groups from sodium to $\mathrm{Cr}^{3+}$-form and precipitating chromium (III) hydroxide into the grain of the cation exchanger with an alkali $\mathrm{NaOH}$ solution. The chromium content in the nanocomposite was determined based on the concentration of counterions released into the solution by direct potentiometry. The surface morphology of hybrid chromium-polymer granules was investigated by scanning electron microscopy. Elemental analysis of the composition of the cleavage of nanocomposite was determined by the energy dispersive X-ray analysis. It was found that the surface of the hybrid granules was uniform. Sulphur and sodium were fairly evenly distributed throughout the entire volume of polymer granules, with the exception of the outer layer depleted in these elements. Chromium was found not only in the surface layer of the granules with the thickness of 10-15 $\mu \mathrm{m}$, but also in the bulk of the grain, where its concentration was significantly lower. The distribution of chromium throughout the grain was uneven. It was shown that the chromium concentration decreased exponentially from the grain periphery to the centre. Elemental mapping confirmed that in the cation exchanger chromium granules are in oxidized form. The mechanism for the formation of a chromium (III) hydroxide nanocomposite - KU-2-8 cation exchanger with sulphonic acid ion exchange groups was proposed. During the ion-exchange saturation reaction the transition of the ion exchanger into the $\mathrm{Cr}^{3+}$-form takes place. A precipitate of chromium (III) hydroxide was localized near the surface of the cation exchanger during the alkalizing of the reaction medium. The formation of $\mathrm{Cr}(\mathrm{OH})_{3}$ precipitate in the nanosized pores of the cation exchanger prevents the diffusion transfer of $\mathrm{Cr}^{3+}$ ions towards its surface, while the Donnan exception does not allow hydroxyl ions in sufficient concentration to penetrate deep enough into the volume of the ion exchanger granule. The possibility of obtaining nanoparticles of a modifier (chromium (III) hydroxide) with an uneven distribution in the volume of granules, combined into micrometer-sized agglomerates, has been shown.
\end{abstract}

Keywords: nanocomposite, cation exchanger KU-2-8, chromium (III) hydroxide

\section{References}

1. Kravchenko T.A., Kalinichev A.I., Polyanskii L.N., Nanokompozity metallionoobmennik, M., Nauka, 2009, 391 p.

2. Huang M., Shen Y., Cheng W., Shao Y. et al, Anal. Chim. Acta., 2005, Vol. 535, Is. 1-2, pp. 5-22.

3. Camargo P.H.C., Satyanarayana K.G., Wypych F, Mater. Res., 2009, Vol. 12, No 1, pp. 1-39.

4. Domènech B., Bastos-Arrieta J., Alonso A., Macanás J., Muñoz M. et al. Bifunctional Polymer-Metal Nanocomposite Ion Exchange Materials, Ion Exchange Technologies, Ayben
Kilislioğlu, IntechOpen, DOI: 10.5772/51579. Available at: https://www.intechopen.com/books/ionexchange-technologies/bifunctional-polymermetal-nanocomposite-ion-exchange-materials

5. Yurova P.A., Karavanova Yu.A., Stenina I.A., Yaroslavtsev A.B., Nanotechnologies in Russia, 2016, Vol. 11, Is. 11-12,pp. 761-765.

6. Safronova E.Yu., Parshina A.V., Yankina K.Yu., Ryzhkova E.A. et al., Membrany i membrannye tekhnologii, 2017, Vol. 7, Is 2, pp. 110116. 
7. Krysanov V.A., Plotnikova N.V., 11. Shel'deshov N.V., Zabolotskii V.I., Kravchenko T.A., Zhurn. fizich. khimii, 2018, Ganych V.V., Elektrokhimiya, 1994, Vol. 30, Is. Vol. 92, Is. 3, pp. 434-438. 12, pp. 1458-1461.

8. Wang D., Lin Z., Wang T., Yao Z. et al., J. 12. Mel'nikov S.S., Shapovalova O.V., Hazard. Mater., 2016, Vol. 308, pp. 328-344. Shel'deshov N.V., Zabolotskii V.I., Membrany $i$ 9. Su C., J. Hazard. Mater., 2017, Vol. 322, membrannye tekhnologii., 2011, Vol. 1, Is. 2, pp. $48-84$.

10. Amrute A.P., Mondelli C., Perez-Ramirez J., Catalysis Science \& Technology, 2012, Vol. 2, pp. 2057-2065.

Козадерова Ольга Анатольевна - доцент кафедры неорганической химии и химической технологии, д.Х.н, Воронежского государственного университета инженерных технологий, Воронеж pp. 149-156.

13. Kozaderova O.A., Nanotechnologies in Russia, 2018, Vol. 13, pp. 508-515.

Kozaderova Olga A. - associate prof., Dr. habil. (chemistry), department of inorganic chemistry and chemical technology, Voronezh State University of Engineering Technologies, Voronezh, email: kozaderova-olga@mail.ru 